\title{
Dihydromyricetin Inhibits Inflammation of Fibroblast-Like Synoviocytes through Regulation of Nuclear Factor- $\kappa$ B Signaling in Rats with Collagen-Induced Arthritis
}

\author{
Jing Wu, ${ }^{1}$ Fu-Tao Zhao, ${ }^{1}$ Kai-Jian Fan, Jun Zhang, Bing-Xing Xu, Qi-Shan Wang, \\ Ting-Ting Tang, and Ting-Yu Wang \\ Departments of Pharmacy (J.W., K.-J.F., B.-X.X., Q.-S.W., T.-Y.W.) and Rheumatology and Immunology (F.-T.Z.), and Shanghai \\ Key Laboratory of Orthopaedic Implants, Department of Orthopaedic Surgery (T.-T.T.), Shanghai Ninth People's Hospital, \\ Shanghai Jiao Tong University School of Medicine, Shanghai, People's Republic of China; and Department of Surgical \\ Oncology, University of Texas MD Anderson Cancer Center, Houston, Texas (J.Z.)
}

Received September 4, 2018; accepted November 26, 2018

\begin{abstract}
Dihydromyricetin (DMY), the main flavonoid of Ampelopsis grossedentata, has potent anti-inflammatory activity. However, the effect of DMY on chronic autoimmune arthritis remains undefined. In this study, we investigated the therapeutic effects of DMY on collageninduced arthritis $(\mathrm{ClA})$. Wistar rats were immunized with bovine type II collagen to establish CIA and were then administered DMY intraperitoneally $(5,25$, and $50 \mathrm{mg} / \mathrm{kg}$ ) every other day for 5 weeks. Paw swelling, clinical scoring, and histologic analysis were assessed to determine the therapeutic effects of DMY on the development of arthritis in CIA rats. The results showed that treatment with DMY significantly reduced erythema and swelling in the paws of CIA rats. Pathologic analysis of the knee joints and peripheral blood cytokine assay results confirmed the antiarthritic effects of DMY on synovitis and inflammation.
\end{abstract}

Fibroblast-like synoviocytes (FLSs) were isolated from the synovium of CIA rats and treated with $10 \mathrm{ng} / \mathrm{ml}$ interleukin (IL)-1 $\beta$. DMY significantly inhibited the proliferation, migration, and inflammation of IL- $1 \beta$-induced FLSs, whereas it significantly increased IL-1 $\beta$-induced FLS apoptosis in a dosedependent manner (6.25-25 $\mu \mathrm{M})$. Moreover, DMY suppressed phosphorylation of $I_{\kappa} \mathrm{B}$ kinase (IKK) and inhibitor of NF- $\kappa \mathrm{B} \alpha$ and subsequently reduced the IL- $1 \beta$-induced nucleus translocation of NF- $\kappa \mathrm{B}$ in FLSs. Through a molecular docking assay, we demonstrated that DMY could directly bind to the Thr9 and Asp88 residues in IKK $\alpha$ and the Asp95, Asn142, and Gln167 residues in IKK $\beta$. These findings demonstrate that DMY could alleviate inflammation in CIA rats and attenuate IL- $1 \beta$-induced activities in FLSs through suppression of $\mathrm{NF}-\kappa \mathrm{B}$ signaling.

\section{Introduction}

Rheumatoid arthritis (RA) is a chronic and systemic autoimmune polyarthritis characterized by progressive exacerbation of multiple joints. There is a massive influx of immune cells into the synovial tissue during the progression of articular destruction in patients, which eventually leads to joint swelling, stiffness, deformity, and dysfunction of the affected joints (Firestein, 2003; McInnes and Schett, 2007; Boissier et al., 2012; van den Brand et al., 2013). In normal synovium, fibroblast-like synoviocytes (FLSs) are responsible for governing the provision of support, nourishment, and lubrication of joint tissue (Karouzakis et al., 2009). However, when the infiltration of several types of immune

This work was supported by the National Natural Science Foundation of China [Grants 81301531, 81572104, and 81874011 (to T.-Y.W.)]. This work was also partially supported by the Shanghai Municipal Science and Technology Commission [Innovation Grant 18140903502 (to T.-Y.W.)]

${ }^{1}$ J.W. and F.-T.Z. contributed equally to this work

https://doi.org/10.1124/jpet.118.253369. cells occurs in the synovial fluid, FLSs become hyperplastic and highly migratory, contributing to the progressive destruction of cartilage and bone. Previous studies reported that FLSs isolated from arthritic mice can invade healthy joints when transferred to nonarthritic mice (Mor et al., 2005; MüllerLadner et al., 2005). In addition, FLSs also release proinflammatory cytokines, proteases, growth factors, and chemokines that recruit and activate peripheral mononuclear cells, neutrophils, macrophages, and mast cells to form an autocrine loop, resulting in extensive cartilage and bone deterioration (Cope, 2008; Drexler et al., 2008; Guma et al., 2010). Thus, FLSs could be a potential target for the treatment of RA. However, there is currently no specific FLS-targeted or FLS-inhibited drug for RA treatment.

Dihydromyricetin (DMY), a type of flavonoid, is the main bioactive component of Ampelopsis grossedentata, which is a widely distributed herbal medicine in Southern China (Okazaki et al., 2005). Previous reports demonstrated that DMY has numerous biologic and pharmacological activities, including antioxidative, anti-inflammatory, antiapoptotic, anticarcinogenic,

ABBREVIATIONS: BSA, bovine serum albumin; CIA, collagen-induced arthritis; Ct, threshold cycle; DMEM, Dulbecco's modified Eagle's medium; DMY, dihydromyricetin; FBS, fetal bovine serum; FLS, fibroblast-like synoviocyte; $l_{\kappa} \mathrm{B} \alpha$, inhibitor of nuclear factor- $\kappa \mathrm{B} \alpha$; IKK, I $\kappa \mathrm{B}$ kinase; IL, interleukin; NF- $\kappa$ B, nuclear factor $\kappa$-light-chain-enhancer of activated B cells; PBS, phosphate-buffered formalin; PCR, polymerase chain reaction; PFA, paraformaldehyde; PI, propidium iodide; RA, rheumatoid arthritis; TNF, tumor necrosis factor. 
and antidiabetic effects (Chen et al., 2015; Li et al., 2015; Ye et al., 2015; Le et al., 2016; Wu et al., 2017). Considering that DMY possesses an anti-inflammatory property, we investigated its potential capacity to inhibit the chronic autoimmune inflammation of RA. Nuclear factor $\kappa$-light-chain-enhancer of activated B cells $(\mathrm{NF}-\kappa \mathrm{B})$ plays a crucial role in the expression of various proinflammatory mediators (McInnes and Schett, 2007; Ivashkiv, 2011). An increasing number of studies have demonstrated that the NF- $\kappa \mathrm{B}$ pathway is one of the key signaling pathways in promoting joint inflammation (Okazaki et al., 2005; Hah et al., 2010) and FLS proliferation (Vincenti et al., 1998; Murphy and Nagase, 2008) in RA. Previous results demonstrated that NF- $\mathrm{B}$ binding is significantly higher in RA synovium compared with that of osteoarthritis (Okazaki et al., 2005). Whether DMY inhibits RA through regulation of the NF- $\kappa \mathrm{B}$ signaling pathway requires further investigation.

Collagen-induced arthritis (CIA) is an autoimmune disease animal model that is mainly characterized by chronic synovitis concomitant with progressive destruction of the joints. The pathologic and immunologic features of CIA are consistent with those of RA (Larsson et al., 2005; Brenner et al., 2007; Nieto et al., 2015). Similar to human RA, CIA displays several clinical symptoms such as the generation of proinflammatory cytokines, synovitis, and erosion of bone (Cho et al., 2007). Thus, CIA is a well established and clinically relevant model of RA (Hegen et al., 2008). In this study, we chose the rat CIA animal model to investigate the antiarthritic effects of DMY on the development and progression of CIA. We aimed to further evaluate whether DMY exerts a suppressive effect on FLS activity through regulation of the NF- $\kappa$ B signaling pathway.

\section{Materials and Methods}

Animals. Male Wistar rats, aged 7 to 8 weeks, were used in the experiments and housed in specific-pathogen-free facilities in pathogenfree conditions. The rats were fed ad libitum with sterile rat-specific diet feed (LAD 0100; Trophy Feed Technology, NanTong, China) and randomly distributed in cages. All experiments were conducted in strict conformance with guidelines adopted from the U.S. National Institutes of Health Guide for the Care and Use of Laboratory Animals and were approved by the Ethics Committee of Shanghai Ninth People's Hospital, Shanghai Jiao Tong University School of Medicine.

Induction of CIA and Drug Administration. Rats were immunized with an emulsion containing $100 \mu \mathrm{g}$ bovine collagen II (Chondrex, Redmond, WA) with an 1-week interval. Collagen II emulsified 1:1 in incomplete Freund's adjuvant (Chondrex) was injected intradermally around the base of the tail on day -7 , and the same emulsion was injected by the same route on day 0 (Larsson et al., 2005; Brenner et al.,2007). Three experimental groups were injected intraperitoneally with 5,25 , and $50 \mathrm{mg} \mathrm{DMY} / \mathrm{kg}$ rat body weight, respectively, and the same administrations were performed on the rats every other day for 5 weeks. Six rats in the normal group were injected with phosphate buffer (PBS) in the same way as those in the model group. Clinical monitoring of the rats was performed twice a week after the second immunization to determine body weight and test the volume of the hind paws. Simultaneously, rats were scored blindly on a scale from 0 to 3 per hind paw (0-12 per rat) as follows (Leavenworth et al., 2013): 0, normal; 1, mild swelling and/or erythema confined to the midfoot or ankle joint; 2 , moderate edematous swelling extending from the ankle to the metatarsal joints; and 3, pronounced swelling encompassing the ankle, foot, and digits. Immunized rats were randomly divided into four groups: a vehicle control group injected with PBS and three DMYtreated groups ( $n=7$ in each group). DMY was of high-purity liquid chromatography grade, the molecular structure of which is $(2 R, 3 R)$ 3,5,7-trihydroxy-2-(3,4,5-trihydroxyphenyl)-2,3-dihydrochromen-4-one, and was $\geq 98 \%$ pure (Sigma-Aldrich, St. Louis, MO, US). DMY was administered intraperitoneally in a dosage of 5,25 , or $50 \mathrm{mg} / \mathrm{kg}$ in $1 \mathrm{ml}$ volume every other day.

Enzyme-Linked Immunosorbent Assay of Inflammatory Cytokines. Peripheral blood was collected in centrifuge tubes after the last administration of DMY and was centrifuged at $3500 \mathrm{rpm}$ for 10 minutes at room temperature with a cooling centrifuge until coagulation. The clear plasma layer was obtained and stored at $-80^{\circ} \mathrm{C}$ in a deep freezer before assaying. Levels of cytokines interleukin (IL)- $1 \beta$, tumor necrosis factor (TNF)- $\alpha$, and IL-17A in the plasma of experimental rats were detected by an enzyme-linked immunosorbent assay kit (Multisciences, Hangzhou, China) according to standard protocols. The optical density was measured using an automatic microplate reader (Infinite M200 PRO NanoQuant; Tecan, Männedorf, Switzerland) at a wavelength of $450 \mathrm{~nm}$. Each sample was tested in duplicate, and concentrations were determined by reference to standard curves, generated by using the optical density values of standard solutions with 1:2 serial dilutions, for estimating cytokine content.

Histopathological Analysis of Joints. On day 34 after the second immunization, the rats were anesthetized and rapidly sacrificed by cervical dislocation. Whole knee joints of the hind legs were carefully separated and fixed in $4 \%$ paraformaldehyde (PFA) for 24 hours. The joints were decalcified in 0.5 M EDTA, dehydrated through an alcohol gradient, embedded in paraffin wax, sectioned at 4- $\mu \mathrm{m}$ thickness in a sagittal plane, and subjected to routine hematoxylin and eosin staining. Sections were photographed with a cool CCD camera attached to a Zeiss Axioskop 2 plus microscope (Carl Zeiss Microimaging). The micrographs were evaluated by two blinded observers (B.-X.X. and Q.-S.W.). To determine the histopathology of the joint, inflammatory activity was observed in the samples with hematoxylin and eosin staining and scored from 0 to 3 according to the following criteria: 1, mild inflammatory infiltration with no synovial lining cell hyperplasia; 2 , moderate infiltration with some synovial lining cell hyperplasia; and 3, severe infiltration with marked synovial lining cell hyperplasia (Buttgereit et al., 2009).

Synovial Cell Culture and Stimulation. Synovial tissue specimens were isolated from rats with CIA and rinsed with PBS two to three times. Then the tissues were minced into small blocks (approximately $1-\mathrm{mm}^{3}$ pieces), transferred to a small culture flask, and tiled uniformly with a spacing of approximately $1 \mathrm{~mm}$. For the air-liquid interface culture, each flask contained a small amount of Dulbecco's modified Eagle's medium (DMEM; Thermo Fisher Scientific, Rockford, IL) with high glucose supplemented with $10 \%$ fetal bovine serum (FBS; Gibco Life Technologies, Carlsbad, CA) and 1\% antibiotics (penicillin and streptomycin) (Thermo Fisher Scientific) at $37^{\circ} \mathrm{C}$ in a humidified thermostatic incubator (Thermo Fisher Scientific) under $5 \%$ carbon dioxide to ensure that pieces adhered to the flask (Liu et al., 2017). After 4-6 hours, an appropriate amount of fresh culture medium was added to the flask to continue the culture, and the medium was totally exchanged every 2 days with the same volume of fresh DMEM. Within 7 days, FLSs could migrate out from the tissue explant and achieve approximately $80 \%$ confluence (Shang et al., 2017); then the tissues were removed, and the liberated FLSs were trypsinized, collected, resuspended, planted for expansion, and used at passages 3-6 (Xiao et al., 2014; Angiolilli et al., 2017).

Identification of FLSs. FLSs that migrated from synovial tissues on days $0,4,5$, and 6 were observed. FLSs were seeded into a sixwell plate with $8 \times 10^{4}$ cells/well and cultured for 24 hours. The cells were fixed with $100 \%$ methanol for 5 minutes, permeabilized with $0.1 \%$ Triton X-100 for 5 minutes, and then blocked with $1 \%$ bovine serum albumin (BSA) in $0.1 \%$ PBS-Tween for 1 hour. Immunofluorescence staining of FLSs was performed with purified anti-Vimentin antibody (Abcam, Cambridge, UK) at a working dilution of 1:250 overnight at $4^{\circ} \mathrm{C}$ and was followed by a further incubation at room temperature for 1 hour with goat anti-rabbit IgG secondary antibody 
(1:300 dilution; Santa Cruz Biotechnology, Dallas, TX). Nuclear DNA was labeled with 4',6-diamidino-2-phenylindole (Sigma-Aldrich) (Fan et al., 2017; Yang et al., 2017). In addition, phenotype identification and purity analysis of FLSs was performed by fluorescence-activated cell sorting analysis (Doss et al., 2016). FLSs were seeded into a six-well plate with $3 \times 10^{5}$ cells/well and collected after cultivation for 24 hours. Cells were then resuspended with $100 \mu$ l fluorescence-activated cell sorting buffer (1\% BSA in $1 \times$ PBS) containing $0.5 \mathrm{mg} / \mathrm{ml}$ fluorescein isothiocyanate-conjugated monoclonal CD90 (also called Thy-1 or Thy1) antibody (Biolegend, San Diego, CA) and incubated at $4^{\circ} \mathrm{C}$ for 30 minutes under dark conditions. Analysis was conducted by flow cytometry (BD LSRFortessa X-20 cell analyzer; BD Biosciences, San Diego, CA).

Cell Proliferation Assay. Cytotoxicity experiments were performed to measure cell proliferation after administration of DMY to synovial cells alone. In general, FLSs were seeded in 96 -well plates (Corning Glassworks, Corning, NY) with 2500 cells/well; 24 hours later, they were randomized into four groups and treated with vehicle or a test compound DMY (3.125-800 $\mu \mathrm{M})$ for 24 and 48 hours. All tests were performed in triplicate, and cell proliferation rates were assessed by the Cell Counting Kit-8 (CCK-8) assay (Dojindo, Kumamoto, Japan) following the manufacturer's recommendation. At each time point, absorbance was measured at $450 \mathrm{~nm}$ using a microplate reader (Shang et al., 2017). To detect the inhibitory effect of DMY on FLSs, cells were stimulated with $10 \mathrm{ng} / \mathrm{ml}$ IL- $\beta$ (R\&D Systems, Minneapolis, MN) to maintain the inflammation condition and then incubated in the presence of DMY at different concentrations.

In Vitro Migration Assay. FLS migration ability was evaluated in a 24-well microchemotaxis chamber (BioCoat; Corning, Shanghai, China) with an 8- $\mu \mathrm{m}$ pore size. DMY and cells in DMEM without FBS were loaded into the upper chambers, and 10\% FBS medium was added to the lower chambers to evaluate the effect of DMY on migration. After 16 hours, a cotton swab was used to remove the remaining cells from the upper surface of the filter, and the cells adhering beneath were fixed in 4\% PFA for 20 minutes and dyed with $0.1 \%$ crystal violet for 30 minutes. Migrated cells were counted in five random fields for each assay at $20 \times$ magnification using light microscopy (Shang et al., 2017). In addition, a wound scratch assay in vitro was adopted to evaluate FLS migration. FLSs were seeded in 60-mm culture dishes (Corning Glassworks) at a density of $5 \times 10^{5}$ cells $/ \mathrm{ml}$; when they reached $90 \%$ confluence, one parallel wound was created using a sterile $200-\mu \mathrm{l}$ pipette tip. The detached cells were washed away with serumfree media, and the cultured FLSs were then treated with DMY (at concentrations of $6.25,12.5$, and $25 \mu \mathrm{M}$ ) without FBS. Photographs were taken at the indicated time points $(0,16,24$, and 48 hours), and migration was quantified by calculating the percentage of the area of FLSs migrating to the scratch with ImageJ software (National Institutes of Health, Bethesda, MD) (Wang et al., 2016).

Flow Cytometric Analysis of Apoptosis. For analysis of apoptosis, FLSs were exposed to several concentrations $(6.25,12.5$, and $25 \mu \mathrm{M})$ of DMY in DMEM for 24 hours. Approximately $5 \times 10^{5}$ cells were collected and stained with an Annexin V-APC (allophycocyanin)/ propidium iodide (PI) apoptosis kit (eBioscience, San Diego, CA) following the manufacturer's protocol and analyzed by flow cytometry (with a BD LSRFortessa X-20 cell analyzer). Early apoptotic cells were defined as Annexin V positive and PI negative in the lower-right quadrant of the graph, whereas late apoptotic cells were positive for both Annexin V and PI in the upper-right quadrant. The percentages in these two quadrants were calculated to represent the total apoptotic rate (Shang et al., 2017; Liu et al., 2017).

Real-Time Polymerase Chain Reaction. After the same treatment as above, FLSs were homogenized in TRIzol reagent (Ambion, Shanghai, China) to extract total cellular RNA, which was subsequently reverse-transcribed to cDNA using the cDNA reverse transcription kit (Takara Bio, Tokyo, Japan) following the standard protocol. Real-time polymerase chain reaction (PCR) primers for TNF- $\alpha$, IL- $1 \beta$, IL-6, and $\beta$-actin were synthesized by Shanghai Sangon Biological Engineering Technology and Services Co., Ltd. (Shanghai, China). The primers were as follows: TNF- $\alpha$ (forward: 5'-ATGGGCTCCCTCTCATCAGT-3'; reverse: 5'-GCTTGGTGGTTTGCTACGAC-3'), IL-1 $\beta$ (forward: 5'-AGCAGCTTTCGACAGTGAGG-3' ${ }^{\prime}$; reverse: 5' -CTCCACGGGCAAGACATAGG-3'), IL-6 (forward: 5'-CTCTCCGCAAGAGACTTCCAG-3'; reverse: 5'-TTCTGACAGTGCATCATCGCT- $3^{\prime}$ ), and $\beta$-actin (forward: $5^{\prime}$-ACGGTC AGGTCATCACTATCG-3'; reverse: 5'-GGCATAGAGGTCTTTACGGATG-3'). Quantitative real-time PCR was performed on a 7500 Real-Time PCR System (Thermo Fisher Scientific) according to the manufacturer's instructions. The cycling program was conducted as follows: predenaturation at $95^{\circ} \mathrm{C}$ for 30 minutes, then 40 cycles of denaturation at $95^{\circ} \mathrm{C}$ for 5 seconds and annealing and extension at $60^{\circ} \mathrm{C}$ for 34 seconds. Target gene expression was determined relatively to the housekeeping gene as a $2^{-\Delta \mathrm{Ct}}$ value, where the $\Delta$ threshold cycle $(\mathrm{Ct})$ was calculated with the comparative $\mathrm{Ct}$ between $\beta$-actin, as the endogenous control, and the target gene (Anderson et al., 2015; Yeo et al., 2016).

Western Blot Analysis. For each experiment, $3 \times 10^{5}$ cells/well were seeded in six-well plates (Corning Glassworks). When subconfluence (approximately 70\%) was reached, the FLSs were stimulated with $10 \mathrm{ng} / \mathrm{ml} \mathrm{IL-} 1 \beta$ at serial time points $(15,30,45$, and 60 minutes) to determine an appropriate time point, and they were then pretreated with serial concentrations of DMY for 24 hours and exposed to $10 \mathrm{ng} / \mathrm{ml} \mathrm{IL-1} \beta$ for the desired time. Total cellular proteins were extracted by adding ice-cold radioimmunoprecipitation assay lysis buffer (Beyotime, Shanghai, China) along with the protease inhibitor phenylmethanesulfonyl fluoride (100 mM; Biosharp, Wuhan, China) along with a phosphatase inhibitor cocktail $(100 \times$; Thermo Fisher Scientific). The FLSs were placed without shaking for 30 minutes; then the cells were scraped off the plate, and the whole-cell lysates were centrifuged at $14,000 \mathrm{rcf}$ for 15 minutes at $4^{\circ} \mathrm{C}$ (Xin et al., 2014). The obtained supernatants were mixed with $5 \times$ protein loading dye (Sangon Biotech, Shanghai, China) with a ratio of 5:1 and boiled for 5 minutes. The blots were probed with primary antibodies (1:1000 dilution; Cell Signaling Technology, Beverly, MA) against NF- $\kappa \mathrm{B}$ p65, phospho-NF- $\kappa \mathrm{B}$ p65, inhibitor of NF- $\kappa \mathrm{B} \alpha(\mathrm{I} \kappa \mathrm{B} \alpha)$, phospho-I $\kappa \mathrm{B} \alpha$, phospho-I $\kappa \mathrm{B}$ kinase (IKK) $\alpha / \beta, \operatorname{IKK} \alpha$, IKK $\beta$, and glyceraldehyde-3phosphate dehydrogenase at $4^{\circ} \mathrm{C}$ overnight, followed by washing with Tris-buffered saline Tween 20 (Epizyme Scientific, Shanghai, China) and incubation with the corresponding secondary antibodies (1:10,000 dilution; Cell Signaling Technology) for 1 hour at room temperature (Zhou et al., 2014; Li et al., 2017). The expression of each signal protein was shown as the ratio of the staining intensity for the target protein to that for glyceraldehyde-3-phosphate dehydrogenase (Qiao et al., 2016). The band intensity was quantified by ImageJ software.

Immunofluorescence Staining. FLSs cultured on confocal dishes were treated in the same way as for Western blotting. Then they were washed and fixed with $4 \%$ PFA for 30 minutes, permeabilized with $1 \%$ Triton X-100 for 30 minutes, blocked with $3 \%$ BSA for 30 minutes at room temperature, and incubated with primary anti-NF- $\kappa$ B-p65 antibody (1:400 dilution; Cell Signaling Technology) overnight at $4^{\circ} \mathrm{C}$ and with $\mathrm{CY} 3$-labeled goat anti-rabbit IgG as secondary antibody (1:500 dilution;

Santa Cruz Biotechnology) at room temperature for 1 hour. The nuclei were visualized using 4',6-diamidino-2-phenylindole (Xin et al., 2014; Li et al., 2017).

Molecular Modeling. DMY and the rat IKK proteins were preprocessed by LigPrep 3.4 software (Schrödinger, New York, NY) and Protein Preparation Wizard software (Schrödinger), respectively. In silico docking was subsequently performed using the standard precision scoring function of Glide 5.5 software (Schrödinger) with default values for other parameters.

\section{Results}

DMY Inhibits CIA Development in Rats. To investigate the therapeutic effects of DMY on autoimmune arthritis, DMY was administered intraperitoneally to CIA rats every 
other day for 5 weeks. Evident swelling, erythema, edema, and joint rigidity confined to the paws of rats in the vehicle-treated group appeared on day 10 and increasing aggravation of symptom was observed in the following week especially reached a plateau on day 18 after strengthened immunization (Fig. 1A). The intensity of arthritis was evaluated in terms of hind paw swelling, weight loss, and clinical score since the day of the second immunization. CIA rats in the placebo group had lower body weight than normal, whereas rats in the experimental groups treated with different doses of DMY had increased body weight first and then gradually returned to normal body weight. In addition, the degrees of swelling and arthritic scores were alleviated dramatically, especially at the high dose of DMY (Fig. 1B). Therefore, the change in clinical symptoms of CIA rats suggests that DMY could inhibit arthritis significantly.

DMY Attenuates Systemic Inflammation in CIA Rats. At the end of follow-up (day 34), levels of proinflammatory cytokines (IL-1 $\beta$, TNF- $\alpha$, and IL-17A) in peripheral blood plasma were detected by an enzyme-linked immunosorbent assay. Compared with those in the vehicle-treated group, levels of detected inflammatory cytokines were reduced in a dose-dependent manner in the groups treated with different doses of DMY (Fig. 2A).

DMY Ameliorates Joint Injury and Inflammatory Infiltration in CIA Rats. The inflamed joints in the vehicle-treated
CIA group showed clear rheumatoid inflammation presenting as synovial hyperplasia with intense inflammatory cell infiltration compared with the joints of normal rats. DMY could ease the deleterious effects of inflammatory arthritis in CIA rats, as demonstrated by histologic analysis (Fig. 2B). Histologic scores for synovitis showed that DMY markedly arrested articular destruction of CIA rats in a dose-dependent manner (Fig. 2C).

DMY Inhibits Cellular Activities in IL-1 $\beta$-Stimulated FLSs. We isolated FLSs from synovial tissues of CIA rats. Cells that migrated out from the tissue explants were shuttle shaped or polygonal and conformed to the morphologic characteristics of fibroblasts (Fig. 3A). Vimentin was positively expressed in $100 \%$ of FLSs (Fig. 3B). CD90 was the molecule that specifically expressed in FLSs, and our results showed that $95.56 \%$ of FLSs stained positive for CD90 (Fig. 3C).

To rule out the possible toxic effects of DMY on FLSs, cells were exposed to increasing concentrations of DMY to detect cell viability in a Cell Counting Kit-8 assay. The results revealed that no notable cytotoxicity to RA FLSs was observed 24 and 48 hours after DMY treatment $(3.125-400 \mu \mathrm{M})$ (Fig. 4A).

The inhibition of DMY on IL-1 $\beta$ (10 ng/ml) induced FLS proliferation, apoptosis, migration, and inflammation was detected in vitro. We found that IL- $1 \beta$ promoted FLS

A

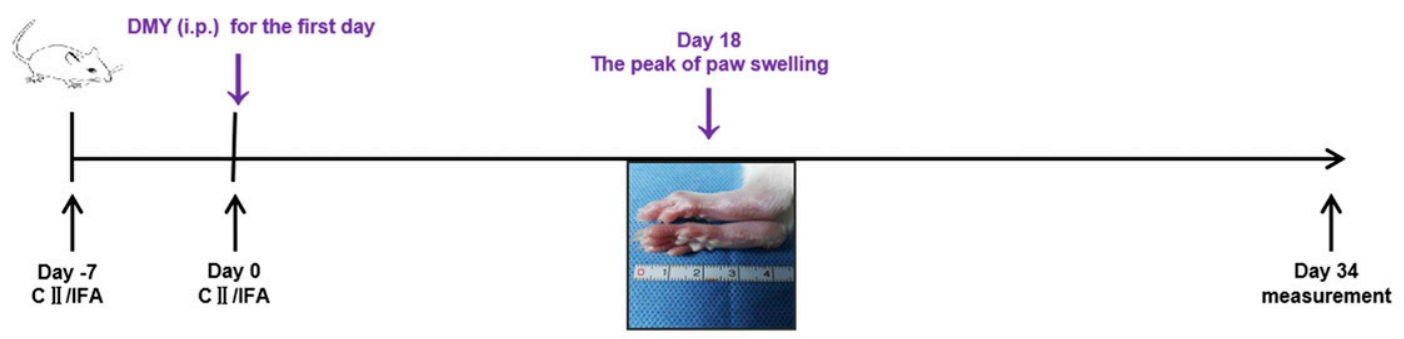

B
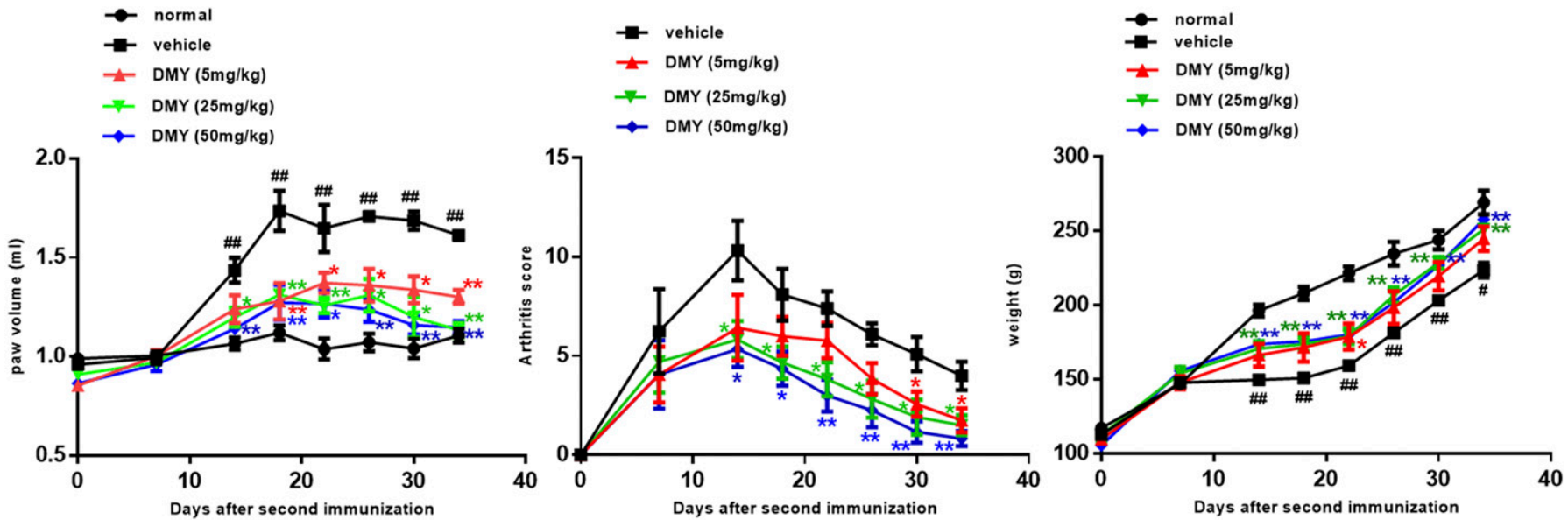

Fig. 1. DMY inhibits development of arthritis in CIA rats. (A) A graphic scheme of CIA induction and DMY administration. Wistar rats were injected with the emulsion or PBS with a 1-week interval as described in the Materials and Methods. After the second injection, rats ( $n=6$ to 7 per group) received DMY for 5 weeks and evaluation was conducted twice a week. (B) Hind-paw swelling of rats was measured by two independent blinded observers (B.-X.X. and Q.-S.W.) from the second injection onward. On day 18, paw swelling in the CIA model reached the peak; from day 14, the value of the DMY-treated group was significantly lower than the vehicle group. The weight of rats in the group injected with DMY vs. vehicle differed significantly, especially in two groups of higher doses. Values are presented as the mean \pm S.E.M. $(n=6$ to 7 rats per group). $* P<0.05 ; * * P<0.01$ vs. the vehicle-treated group; ${ }^{\#} P<0.05 ;{ }^{\# \#} P<0.01$ vs. the normal group. CII, collagen II; IFA, incomplete Freund's adjuvant. 
A

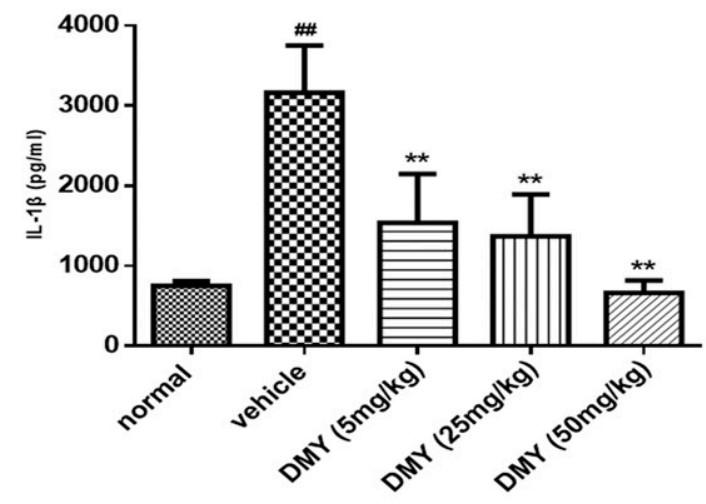

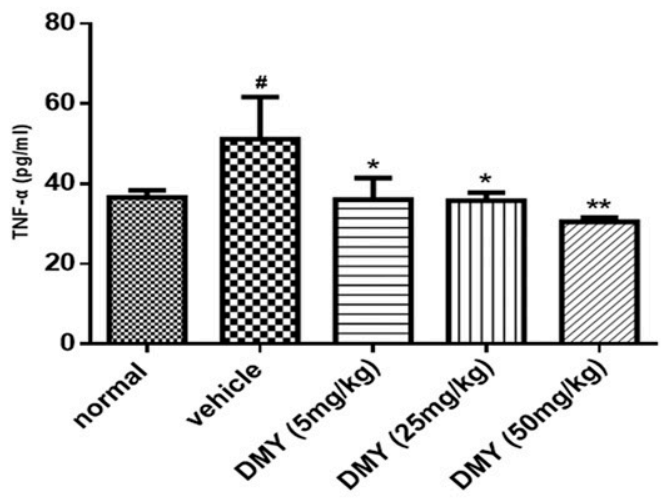
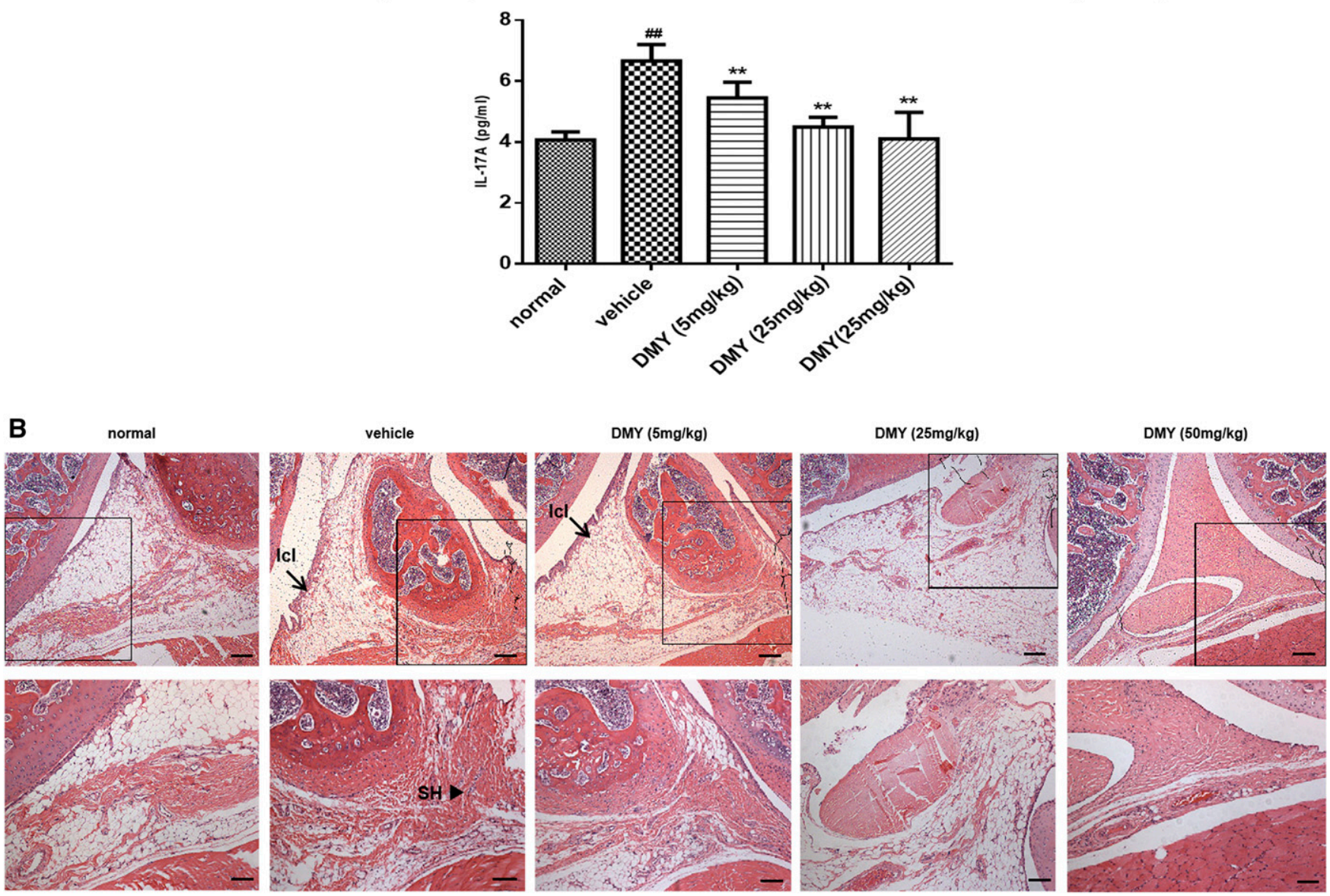

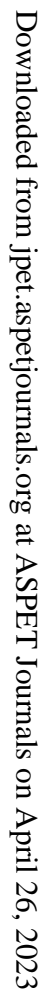

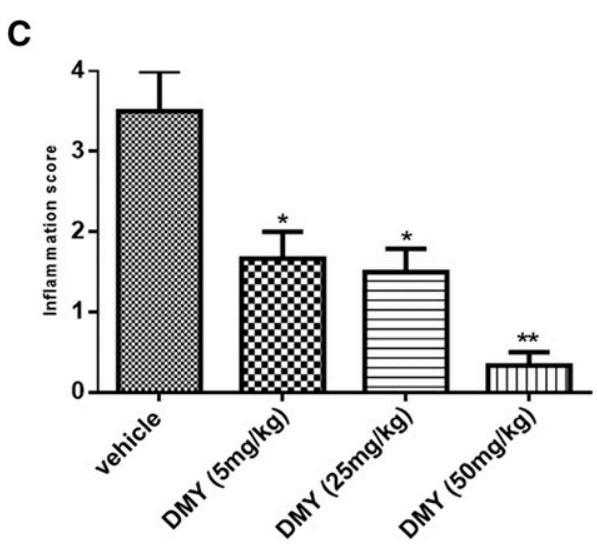

Fig. 2. DMY suppresses inflammation in CIA rats. (A) Plasma cytokine levels. Cytokine levels were quantified in supernatants of peripheral blood of CIA rats isolated on day 34 by the enzyme-linked immunosorbent assay. The plasma of rats administered DMY in vivo contained significantly less IL- $1 \beta$, TNF- $\alpha$, and IL-17A than that of untreated CIA rats. (B) Representative joint sections stained with hematoxylin and eosin. The black boxes represent 
A
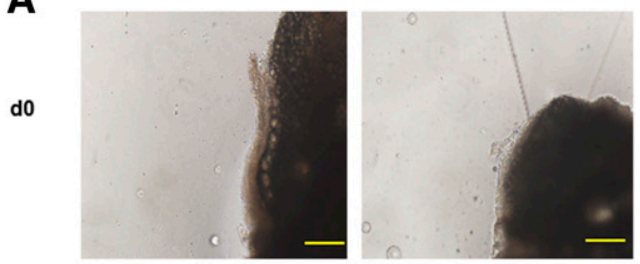

d4

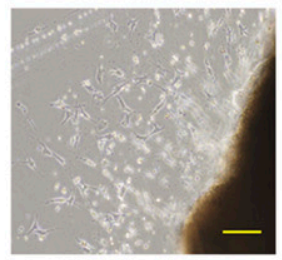

d5

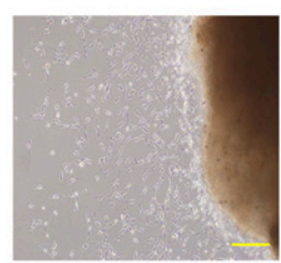

d6

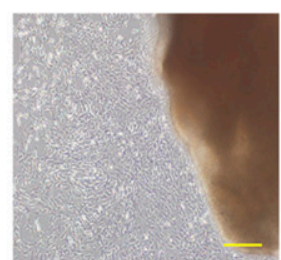

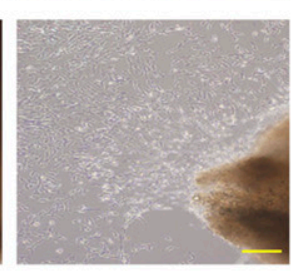

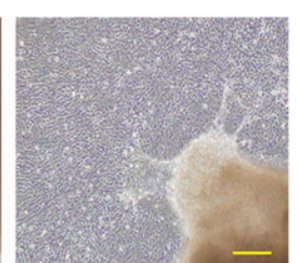

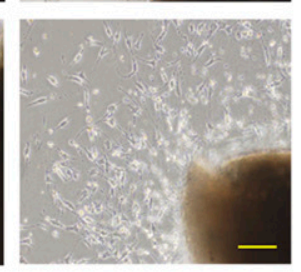

B

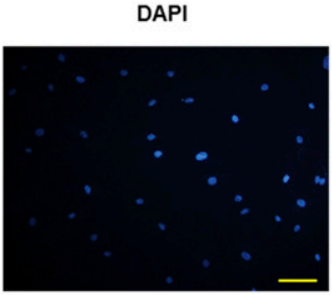

vimentin

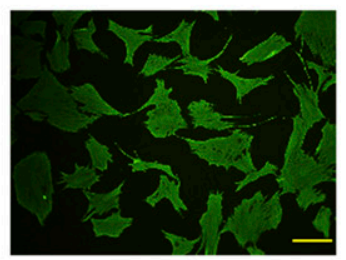

merge

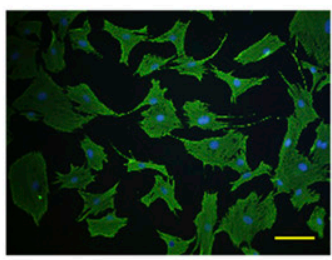

C

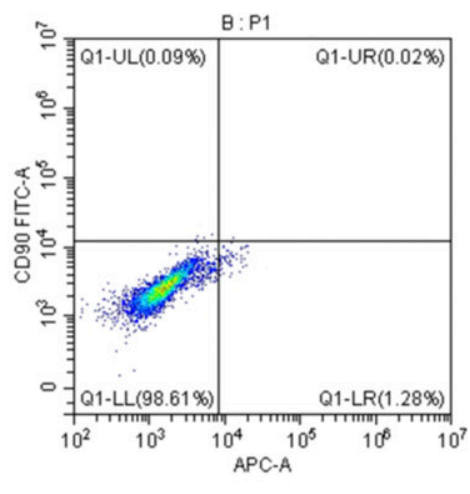

Control (unstained cells)

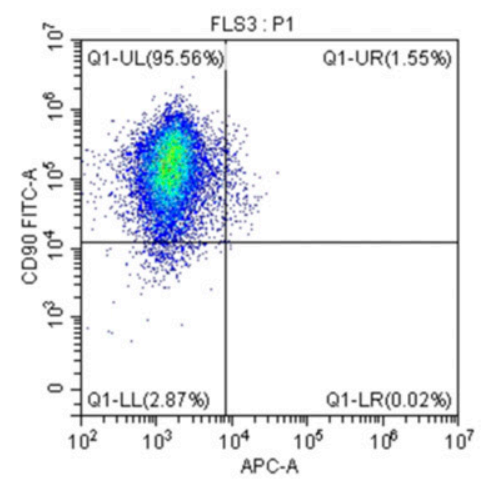

CD90 FITC

Fig. 3. Phenotype and purity analysis of FLSs. (A) Representative images of FLSs migrated from synovial tissues on days 0, 4, 5, and 6. (B) Immunofluorescence images of FLSs stained with vimentin (green) are shown. As presented in the images, vimentin (found in connective tissue and cytoskeleton) was nearly expressed in all FLSs. Scale bar, $10 \mu \mathrm{m}$. (C) Fluorescence-activated cell sorting analysis of FLSs stained with FITC-conjugated CD90 antibody. Representative images are presented from three independent experiments. DAPI, 4',6-diamidino-2-phenylindole; FITC, fluorescein isothiocyanate.

proliferation, whereas DMY abrogated the proliferation dose dependently (Fig. 4B). Annexin V/PI double staining was performed to detect the proapoptotic effects of different concentrations of DMY on IL-1 $\beta$-stimulated FLSs (Fig. 4C). Counting the rates of Annexin V/PI double-positive cells revealed that IL-1 $\beta$ strikingly inhibited FLSs apoptosis; in contrast, the level of cell apoptosis significantly increased with increasing concentrations of DMY (Fig. 4D). We next investigated whether DMY suppresses the migratory capacity of FLSs with a transwell assay and a wound closure assay. First, we determined that the appropriate period of migration was 16 hours (Fig. 5, A and B). Treatment with IL- $1 \beta$ significantly enhanced cell motility, causing a 2 - to 3 -fold increase in migrated cell numbers compared with that in the placebo group in the transwell assay. In contrast, treatment with DMY counteracted this trend and evidently abrogated IL- $1 \beta$-mediated FLS migration (Fig. 5, C and D). Consistent with the above results, the erosion trace assay showed the same tendency (Fig. 5E) and DMY possessed suppressive activity in a concentrationdependent manner in the wound scratch assay (Fig. 5F). Since inflammation is obvious in RA FLSs, it is reasonable to determine the effect of DMY on the expression of proinflammatory cytokines in FLSs. We evaluated the mRNA levels of several major inflammatory cytokines such as IL-6, TNF- $\alpha$, and IL- $1 \beta$ after exposure to different concentrations of DMY for 24 hours. Compared with those in the control group, reduced mRNA levels of these cytokines were observed in FLSs treated with different concentrations of DMY (Fig. 6A). Collectively, consistent with the previous results of the capacity of DMY on synovitis in vivo, DMY could substantially restrain the activity of IL- $1 \beta$-stimulated FLSs, providing a potential therapeutic effect of DMY on synovitis of rheumatoid arthritis.

DMY Inhibits FLSs through Regulation of NF- $\mathrm{kB}$ Signaling. Compared with that in the control medium, the phosphorylation of $\mathrm{NF}-\kappa \mathrm{B}$ was apparently increased in IL- $1 \beta$-induced FLSs. In addition, a time-course analysis revealed that, under IL- $1 \beta$ stimulation for 15 minutes, activated IKK could promote the phosphorylation and proteolytic degradation of $\mathrm{I} \kappa \mathrm{B} \alpha$ and the phosphorylation of NF- $\kappa \mathrm{B}$, contributing to the translocation of NF- $\kappa \mathrm{B}$ to the nucleus (Fig. $6 \mathrm{~B}$ ). We further examined the effect of DMY on the NF- $\kappa \mathrm{B}$ pathway in cultured FLSs. As shown in Fig. 6C, pretreatment with DMY markedly inhibited 
A

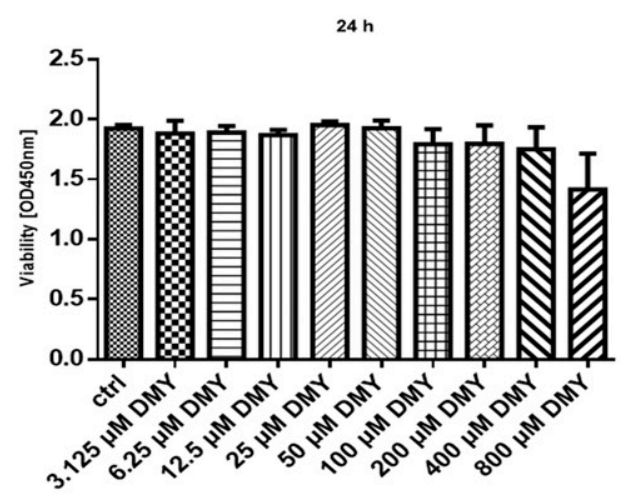

B

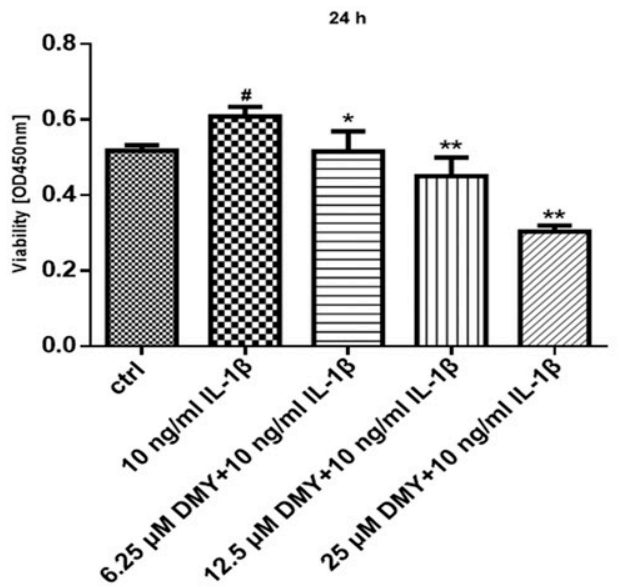

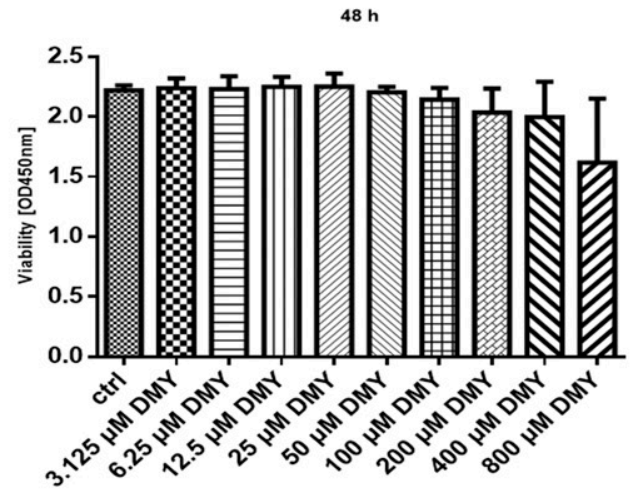

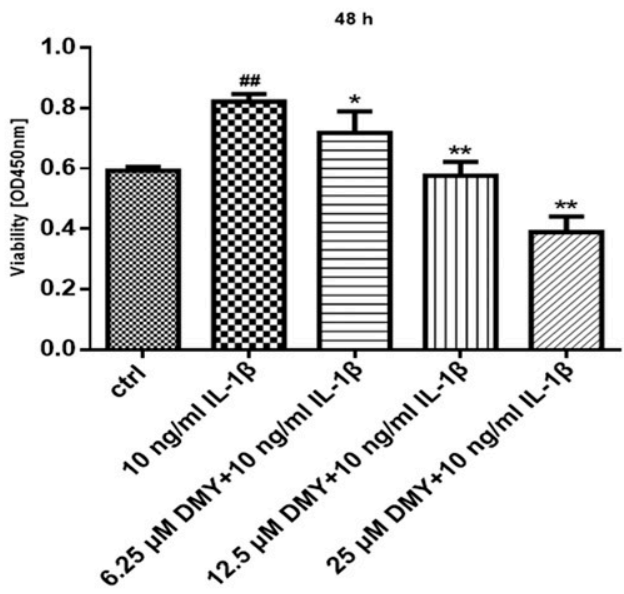

C
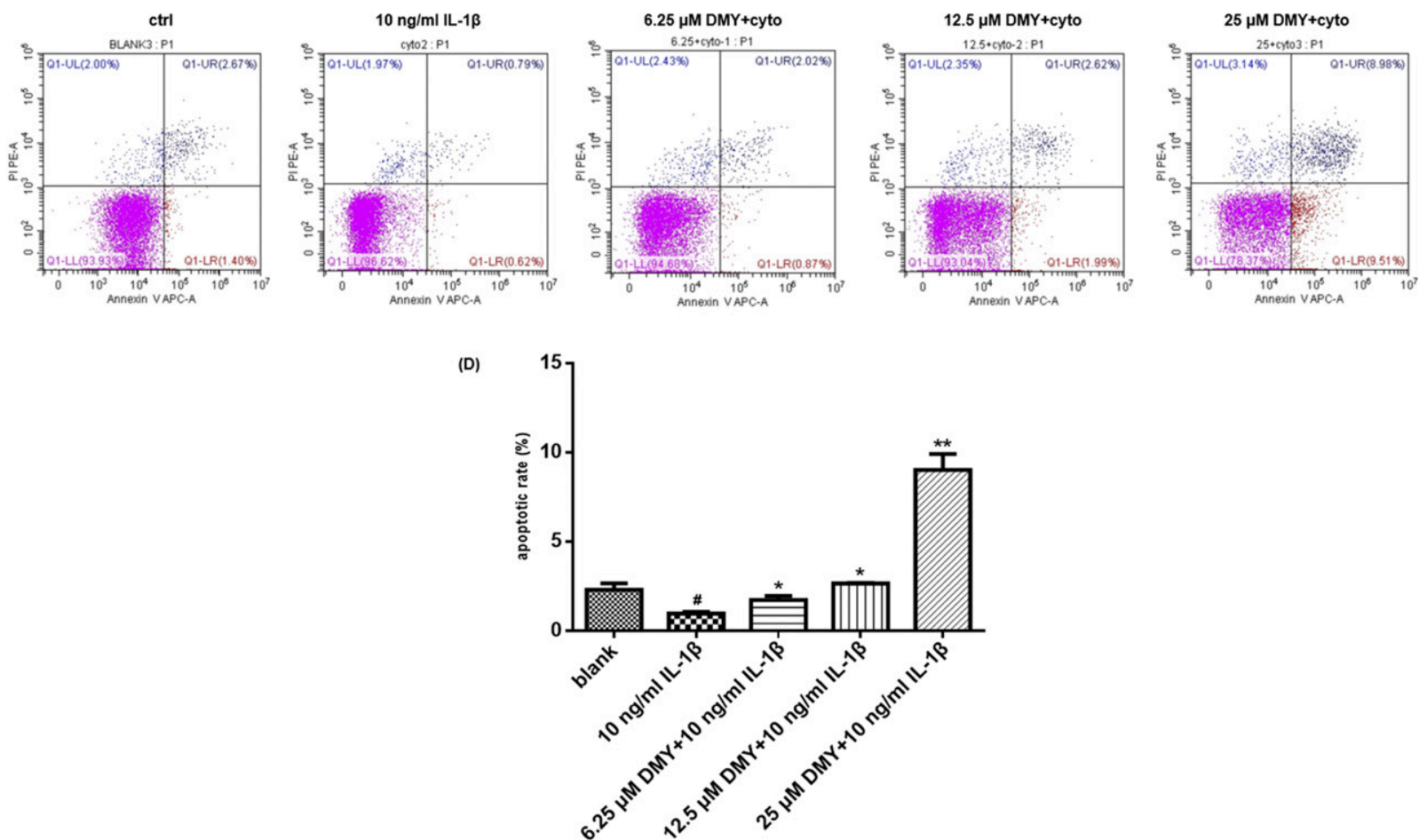

Fig. 4. DMY regulates IL- $1 \beta$-induced FLS proliferation and apoptosis. (A) FLSs were treated with a series of concentrations of DMY for 24 and 48 hours and results showed that there was no cytotoxicity of DMY. (B) When FLSs were exposed to DMY for 24 and 48 hours with the stimulation of $10 \mathrm{ng} / \mathrm{ml}$ IL-1 $\beta$, the FLSs in DMY-treated groups showed evidently lower proliferation ability than those in vehicle-treated groups. (C) FLSs were incubated with DMY (6.25, 12.5 , and $25 \mu \mathrm{M}$ ) for 24 hours after stimulation of $10 \mathrm{ng} / \mathrm{ml} \mathrm{IL-1 \beta}$. Cell apoptosis was determined by Annexin V/PI staining. Populations in the upper-right quadrant of the flow cytometric graph represent apoptotic cells. (D) The percentage of apoptotic cells was quantified. All values represent the mean \pm S.E.M. of three independent experiments. ${ }^{*} P<0.05$; ${ }^{*} P<0.01$ vs. the vehicle-treated group; ${ }^{\sharp} P<0.05$; ${ }^{\# \#} P<0.01$ vs. the normal group. OD, optical density. 
A

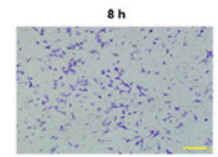

$16 \mathrm{~h}$

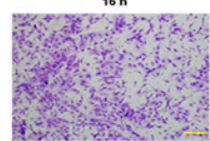

$24 \mathrm{~h}$
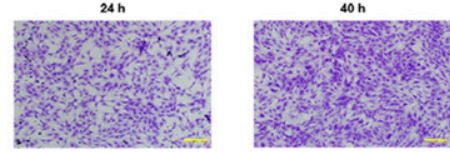

B
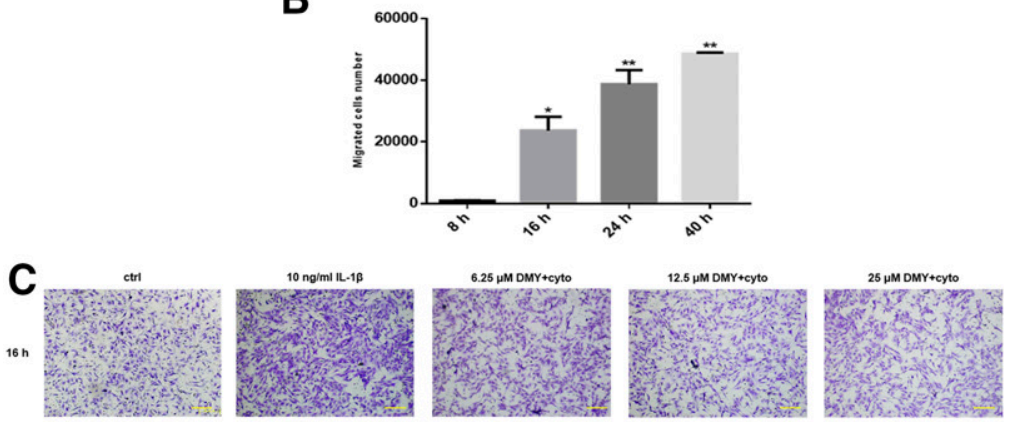

D

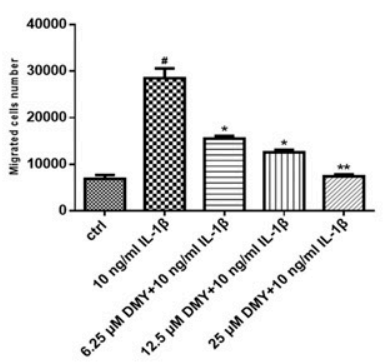

$\mathbf{E}$
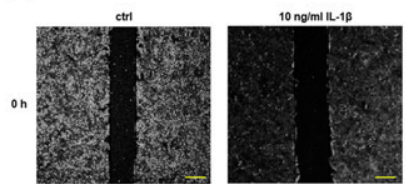

6.25 uM DMYrecyto
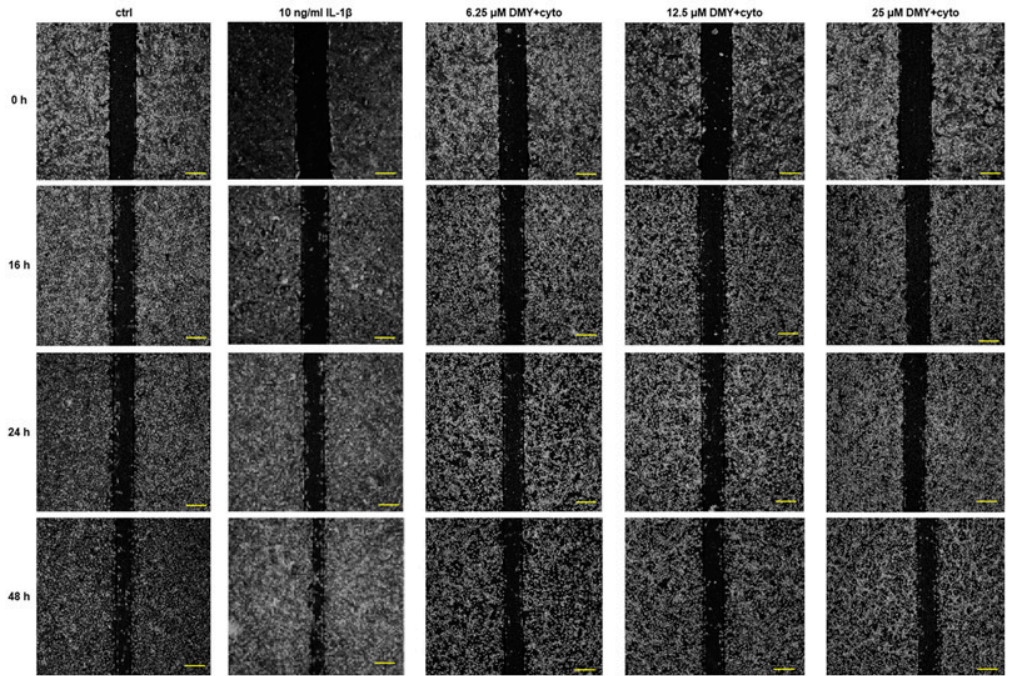

$\mathbf{F}$

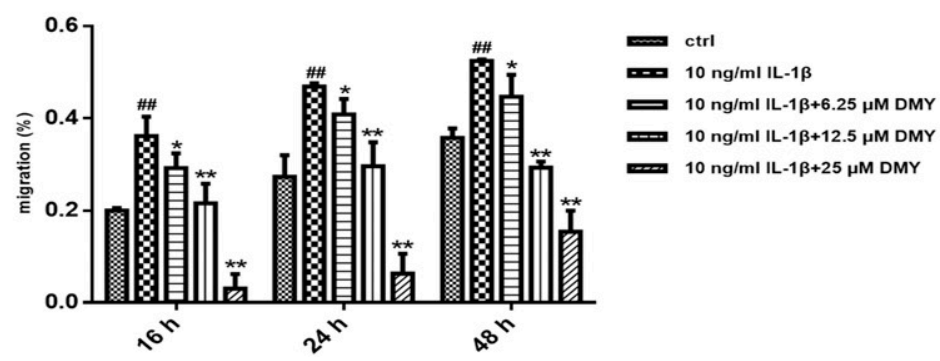

Fig. 5. DMY inhibits migration of IL- $1 \beta$-induced FLSs. (A and C) Evaluation of the Boyden chamber experiment. FLSs were added to the upper chambers of transwells. Representative images of three independent experiments are shown. Scale bar is $10 \mu \mathrm{m}$. (B) Cell migration was quantified by counting migrated cells in nine microscope fields. The period of migration was determined at the time of 16 hours. Values are the mean \pm S.E.M. of at least three independent experiments. $* P<0.05$; $* * P<0.01$ vs. the time point at 8 hours. (D and F) DMY dose-dependently attenuated cell migration. (E) Wound migration of FLSs derived from CIA rats. Representative photomicrographs of a scratch assay showed that the similar trend as the transwell assay. Scale bar, $25 \mu \mathrm{m}$. Values are the mean \pm S.E.M. of at least three independent experiments. $* P<0.05 ; * * P<0.01 \mathrm{vs}$. the vehicle-treated group; ${ }^{\#} P<0.05 ;{ }^{\# \#} P<0.01$ vs. the normal group.
IL- $1 \beta$-mediated phosphorylation of IKK, p65, and $\mathrm{I} \kappa \mathrm{B} \alpha$ in a concentration-dependent manner. These results were further confirmed by immunofluorescence staining using an anti-p65 antibody targeted to the major subunit of NF- $\kappa \mathrm{B}$ to evaluate the effect of DMY on NF- $\kappa \mathrm{B}$ activation and translocation. We found that DMY significantly reduced p65 nuclear 


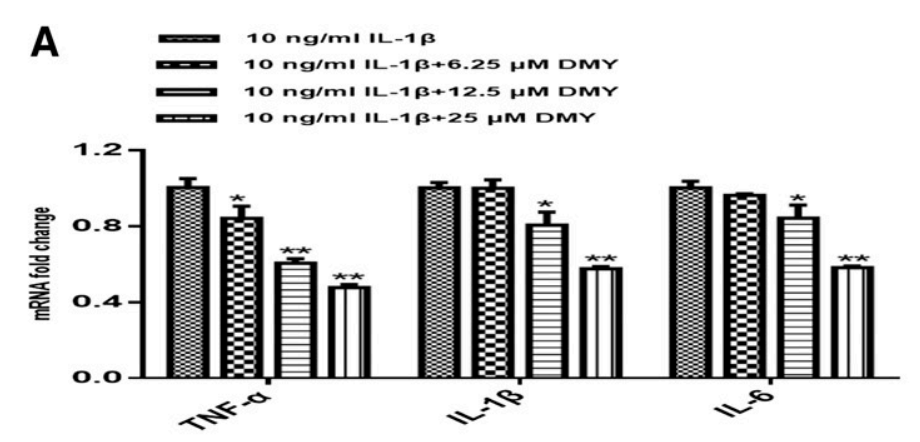

B
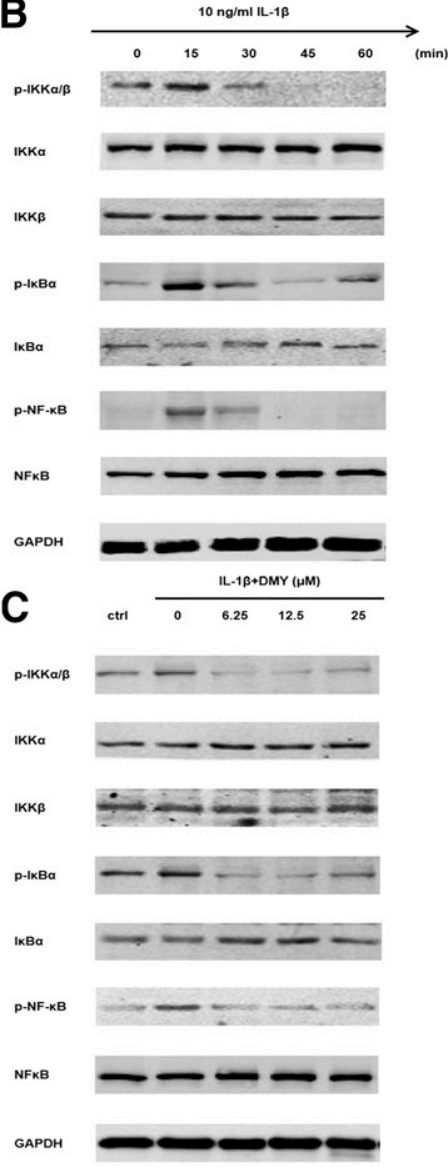

E

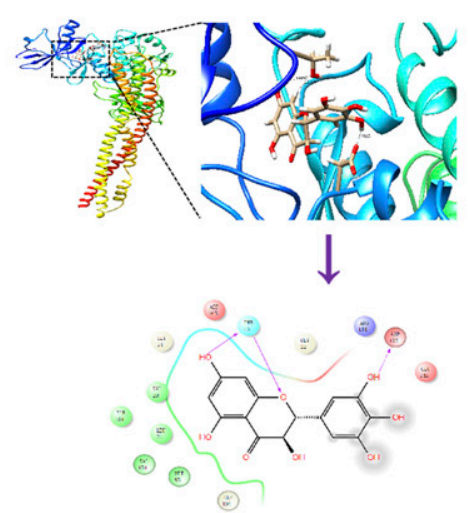

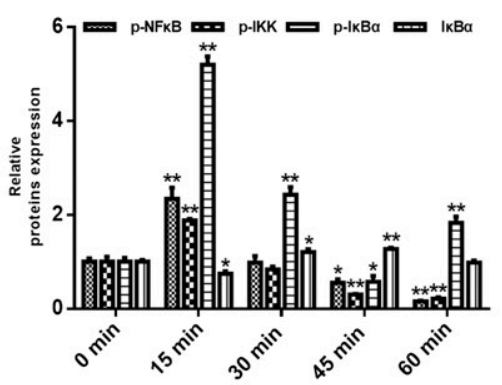

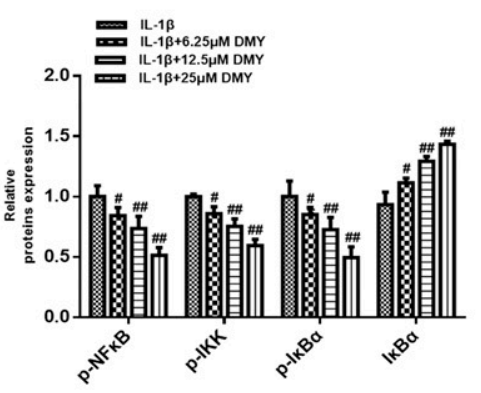

ІКк-в
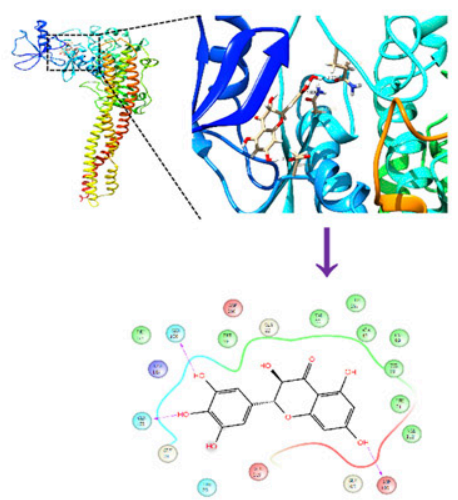

Fig. 6. DMY regulates NF- $\kappa \mathrm{B}$ signaling in FLSs. Cultured FLSs were stimulated with $10 \mathrm{ng} / \mathrm{ml}$ IL-1 $\beta$. (A) DMY diminishes production of inflammatory cytokines in FLSs, especially in higher doses. mRNA levels of cytokines were normalized to $\beta$-actin, and the results are presented as the fold decrease above IL-1 $\beta$ treatment (no DMY treatment). ${ }^{*} P<0.05$; $* * P<0.01$ vs. IL-1 $\beta$ stimulation. (B) Protein levels of phospho-IKK, phospho$\mathrm{p} 65$, and phospho-I $\kappa \mathrm{B}$ or total proteins were determined using Western blotting. With IL- $1 \beta$ stimulation $(10 \mathrm{ng} / \mathrm{ml})$ for the indicated time points to ensure that 15 minutes is the optimal time point for the maximal activation of $\mathrm{NF}-\kappa \mathrm{B}$ signaling. $* P<$ $0.05 ; * * P<0.01$ compared with the untreated control. (C) Thus, in coincubation experiments, FLSs were treated with DMY for 24 hours prior to stimulation with IL-1 $\beta$ for 15 minutes. ${ }^{\#} P<$ 0.05 ; ${ }^{\# \#}<0.01$ compared with IL- $1 \beta$. (D) Effect of DMY on nuclear translocation of p65 in FLSs. Representative immunofluorescence photographs are shown, as the staining of p65 localization is green and nuclei stained with DAPI is blue. (E) Molecular docking of IKK $\alpha$ and $\operatorname{IKK} \beta$ with DMY. All values represent the mean \pm S.E.M. of three independent experiments. DAPI, 4',6-diamidino-2-phenylindole; GAPDH, glyceraldehyde3 -phosphate dehydrogenase. translocation in IL-1 $\beta$-induced FLSs (Fig. 6D). The molecular docking assay demonstrated that DMY directly bound to the Thr9 and Asp88 residues in IKK $\alpha$ and the Asp95, Asn142, and
Gln167 residues in IKK $\beta$ (Fig. 6E). Collectively, our findings suggest that DMY suppressed IL- $1 \beta$-induced phosphorylation of IKK, degradation of $\mathrm{I} \kappa \mathrm{B} \alpha$, and nuclear translocation of 
NF- $\kappa \mathrm{B}$ in FLSs by directly binding to the specific amino acid residues of IKK.

\section{Discussion}

The etiopathogenesis of RA is considered as multiple joint damage caused by abnormal immune regulation, in which synovitis is the typical pathologic feature. Inflammation, loss of synovial homeostasis, and progressive synovium-mediated joint destruction are typical features of inflammatory arthritis (Karouzakis et al., 2009). The synovium in RA manifests hyperplastic and invasive properties mediated by local lymphocytes in synovial tissue (Takemura et al., 2001). In addition, the balance between proteases and their inhibitors in normal synovium is broken by the excessive expression of matrix metalloproteinases that eventually leads to the destruction of cartilage and bone erosion (García-Vicuña et al., 2004). In our study, we found that the paw volume and arthritis score of CIA rats progressively decreased, and body weight gradually increased with DMY administration (Fig. 1). The systemic inflammation mediated by cytokines (including TNF- $\alpha$, IL- $1 \beta$, and IL-17A) in peripheral blood was substantially suppressed by treatment with DMY. Moreover, the results of histologic analysis of knee joints in the animals administered different doses of DMY showed decreased inflammatory cell infiltration and alleviated synovial hyperplasia. Taken together, our results demonstrate that DMY could evidently abrogate synovitis and systemic inflammation in CIA rats.

Synovial cells contain type A or macrophage-like synovial cells and type B or FLSs, of which type A cells possess little capacity to proliferate (Bartok and Firestein, 2010). FLS hyperplasia in RA displays surprisingly pathogenic behavior to promote pannus growth, inflammation, and destruction of the joint (Bottini and Firestein, 2013) and thus to serve as the link between immune response and joint damage ( $\mathrm{Qu}$ et al., 1994a). Thus, we focus on the inhibitory effects of DMY on arthritis by specifically targeting FLSs, which provide nourishment, support, and lubrication to the joint in the normal synovium and become highly hyperplastic and invasive in the inflammatory milieu. Since IL-1 $\beta$ levels in peripheral blood were significantly increased in CIA rats and IL- $1 \beta$ plays a key role in synovitis and joint destruction in patients with RA (Tak and Bresnihan, 2000), we chose IL- $1 \beta$ as the stimulatory cytokine in vitro experiments to maintain the inflammatory state and invasive activity of FLSs similar to in vivo status of RA. Previous results demonstrated that synovitis is mainly mediated by FLSs, which possess the ability to secrete proinflammatory cytokines as well as mediators of bone destruction such as matrix metalloproteinases and the receptor activator of NF- $\kappa$ B ligand, attracting and activating proinflammatory cells in the inflamed joint (Bottini and Firestein, 2013; Goldring et al., 2013) and ultimately resulting in destruction of the affected joints (Choy and Panayi, 2001; Rico et al., 2007). We evaluated the mRNA levels of several major inflammatory cytokines such as IL-6, TNF- $\alpha$, and IL- $1 \beta$ after exposure to different concentrations of DMY in IL- $1 \beta$-stimulated FLSs. Compared with those in the control group, reduced mRNA levels of these cytokines were observed in DMY groups dose dependently (Fig. 6A). The migratory capacity of FLSs was detected with transwell and wound-healing assays. DMY evidently abrogated the IL- $1 \beta$-mediated FLS migration in a concentrationdependent manner (Fig. 5). Synovial hyperplasia is caused by restrained apoptosis concomitant with promoted proliferation of FLSs (Liu and Pope, 2003). The results showed that abnormalities in FLS proliferation and apoptosis in RA, similar to tumor cells, may be caused by inactivation of p53, leading to defects in antiproliferative and proapoptotic effects, (Pap et al., 2004; Lefêvre et al., 2009). We found that DMY exerts a potent suppressive effect on IL-1 $\beta$-mediated FLS proliferation, while promoted function on IL- $1 \beta$-mediated FLS apoptosis. Moreover, the cell cytotoxicity assay showed no differences in cell viability between the different doses of DMY groups $(6.25-25 \mu \mathrm{M})$ and the vehicle control group. Thus, DMY may attenuate the arthritis of CIA rats by regulating the activities of inflammation, migration, proliferation, and apoptosis of inflammatory FLSs.

The NF- $\kappa$ B pathway has been demonstrated to be highly activated in conventional inflammatory diseases (Miagkov et al., 1998; Tak and Bresnihan, 2000). NF- $\kappa$ B normally combines with $\mathrm{I} \kappa \mathrm{B} \alpha$ in cytoplasm and can be released after the phosphorylation of $\mathrm{I} \kappa \mathrm{B} \alpha$, which is mediated by the Separation of IKK $\beta$ with IKK $\alpha$ in response to a cellular stimulation like proinflammatory cytokines (Bartok and Firestein, 2010). The IKK complex of $\mathrm{IKK} \alpha$ and $\operatorname{IKK} \beta$ is a convergence point for NF- $\kappa \mathrm{B}$ signaling (Bartok and Firestein, 2010). The liberated NF- $\kappa \mathrm{B}$ migrates into the nucleus to regulate the transcriptional activity of the target genes and precedes the deterioration of synovial inflammation in RA (Tak and Bresnihan, 2000). Previous studies also revealed that DMY can decrease the quantity of the receptor activator of $\mathrm{NF}-\kappa \mathrm{B}$ ligand to suppress activation of NF- $\kappa \mathrm{B}$ and phosphorylation of p38, resulting in lowered serum levels of inflammatory cytokines in pharyngitis and decreased bone loss in osteoporosis (Hou et al., 2015; Zhao et al., 2017). Here, we demonstrated that DMY significantly attenuates the IL- $1 \beta$-induced phosphorylation of IKK and $\mathrm{I}_{\kappa} \mathrm{B} \alpha$, thereby decreasing NF- $\kappa \mathrm{B}$ translocation into the nucleus and consequently regulating the transactivation of NF- $\kappa \mathrm{B}-$ dependent genes such as proinflammatory cytokines. Molecular docking of IKK $\alpha$ and IKK $\beta$ with DMY further demonstrated that DMY could downregulate NF- $\kappa$ B signaling by direct binding to IKK, thus inhibiting IKK phosphorylation.

Overall, this study clearly demonstrates that DMY exerts an antiarthritic effect in CIA rats through regulation of NF- $\kappa \mathrm{B}$ signaling of FLSs. Our findings suggest that DMY may serve as a potential key therapeutic agent for RA treatment.

\section{Authorship Contributions}

Participated in research design: Wu, T.-Y. Wang.

Conducted experiments: Wu, Fan, Xu, Zhao, Q. S. Wang.

Contributed new reagents or analytic tools: Wu, Zhang, Tang, T.-Y. Wang.

Performed data analysis: Wu, T.-Y. Wang.

Wrote or contributed to the writing of the manuscript: $\mathrm{Wu}$, Zhao, T.-Y. Wang.

\section{References}

Anderson AE, Pratt AG, Sedhom MA, Doran JP, Routledge C, Hargreaves B, Brown PM, Lê Cao KA, Isaacs JD, and Thomas R (2015) IL-6-driven STAT signalling in circulating $\mathrm{CD} 4^{+}$lymphocytes is a marker for early anticitrullinated peptide antibody-negative rheumatoid arthritis. Ann Rheum Dis 75:466-473.

Angiolilli C, Kabala PA, Grabiec AM, Van Baarsen IM, Ferguson BS, García S, Malvar Fernandez B, McKinsey TA, Tak PP, Fossati G, et al. (2017) Histone deacetylase 3 regulates the inflammatory gene expression programme of rheumatoid arthritis fibroblast-like synoviocytes. Ann Rheum Dis 76:277-285.

Bartok B and Firestein GS (2010) Fibroblast-like synoviocytes: key effector cells in rheumatoid arthritis. Immunol Rev 233:233-255.

Boissier MC, Semerano L, Challal S, Saidenberg-Kermanac'h N, and Falgarone G (2012) Rheumatoid arthritis: from autoimmunity to synovitis and joint destruction. $J$ Autoimmun 39:222-228.

Bottini N and Firestein GS (2013) Duality of fibroblast-like synoviocytes in RA: passive responders and imprinted aggressors. Nat Rev Rheumatol 9:24-33. 
Brenner M, Laragione T, Mello A, and Gulko PS (2007) Cia25 on rat chromosome 12 regulates severity of autoimmune arthritis induced with pristane and with collagen. Ann Rheum Dis 66:952-957.

Buttgereit F, Zhou H, Kalak R, Gaber T, Spies CM, Huscher D, Straub RH, Modzelewski J, Dunstan CR, and Seibel MJ (2009) Transgenic disruption of glucocorticoid signaling in mature osteoblasts and osteocytes attenuates $\mathrm{K} / \mathrm{BxN}$ mouse serum-induced arthritis in vivo. Arthritis Rheum 60:1998-2007.

Chen S, Zhao X, Wan J, Ran L, Qin Y, Wang X, Gao Y, Shu F, Zhang Y, Liu P, et al. (2015) Dihydromyricetin improves glucose and lipid metabolism and exerts antiinflammatory effects in nonalcoholic fatty liver disease: a randomized controlled trial. Pharmacol Res 99:74-81.

Cho YG, Cho ML, Min SY, and Kim HY (2007) Type II collagen autoimmunity in a mouse model of human rheumatoid arthritis. Autoimmun Rev 7:65-70.

Choy EH and Panayi GS (2001) Cytokine pathways and joint inflammation in rheumatoid arthritis. $N$ Engl $J$ Med 344:907-916.

Cope AP (2008) T cells in rheumatoid arthritis. Arthritis Res Ther 10 (Suppl 1):S1.

Doss HM, Ganesan R, and Rasool M (2016) Trikatu, an herbal compound ameliorates rheumatoid arthritis by the suppression of inflammatory immune responses in rats with adjuvant-induced arthritis and on cultured fibroblast like synoviocytes via the inhibition of the $\mathrm{NF}_{\kappa} \mathrm{B}$ signaling pathway. Chem Biol Interact 258:175-186.

Drexler SK, Kong PL, Wales J, and Foxwell BM (2008) Cell signalling in macrophages, the principal innate immune effector cells of rheumatoid arthritis. Arthritis Res Ther 10:216.

Fan P, He L, Hu N, Luo J, Zhang J, Mo LF, Wang YH, Pu D, Lv XH, Hao ZM, et al. (2017) Effect of 1,25-(OH)2D3 on proliferation of fibroblast-like synoviocytes and expressions of pro-inflammatory cytokines through regulating microRNA-22 in a rat model of rheumatoid arthritis. Cell Physiol Biochem 42:145-155.

Firestein GS (2003) Evolving concepts of rheumatoid arthritis. Nature 423:356-361.

García-Vicuña R, Gómez-Gaviro MV, Domínguez-Luis MJ, Pec MK, González-Alvaro I, Alvaro-Gracia JM, and Díaz-González F (2004) CC and CXC chemokine receptors mediate migration, proliferation, and matrix metalloproteinase production by fibroblast-like synoviocytes from rheumatoid arthritis patients. Arthritis Rheum 50:3866-3877.

Goldring SR, Purdue PE, Crotti TN, Shen Z, Flannery MR, Binder NB, Ross FP, and McHugh KP (2013) Bone remodelling in inflammatory arthritis. Ann Rheum Dis 72 (Suppl 2):ii52-ii55.

Guma M, Kashiwakura J, Crain B, Kawakami Y, Beutler B, Firestein GS, Kawakami T, Karin M, and Corr M (2010) JNK1 controls mast cell degranulation and IL-1beta production in inflammatory arthritis. Proc Natl Acad Sci USA 107:22122-22127.

Hah YS, Lee YR, Jun JS, Lim HS, Kim HO, Jeong YG, Hur GM, Lee SY, Chung MJ, Park JW, et al. (2010) A20 suppresses inflammatory responses and bone destruction in human fibroblast-like synoviocytes and in mice with collagen-induced arthritis. Arthritis Rheum 62:2313-2321.

Hegen M, Keith JC Jr., Collins M, and Nickerson-Nutter CL (2008) Utility of animal models for identification of potential therapeutics for rheumatoid arthritis. Ann Rheum Dis 67:1505-1515.

Hou XL, Tong Q, Wang WQ, Shi CY, Xiong W, Chen J, Liu X, and Fang JG (2015) Suppression of inflammatory responses by dihydromyricetin, a flavonoid from Ampelopsis grossedentata, via inhibiting the activation of NF-кB and MAPK signaling pathways. J Nat Prod 78:1689-1696.

Ivashkiv LB (2011) Inflammatory signaling in macrophages: transitions from acute to tolerant and alternative activation states. Eur J Immunol 41:2477-2481.

Karouzakis E, Gay RE, Gay S, and Neidhart M (2009) Epigenetic control in rheumatoid arthritis synovial fibroblasts. Nat Rev Rheumatol 5:266-272.

Larsson E, Harris HE, Palmblad K, Månsson B, Saxne T, and Klareskog L (2005) CNI-1493, an inhibitor of proinflammatory cytokines, retards cartilage destruction in rats with collagen induced arthritis. Ann Rheum Dis 64:494-496.

Le L, Jiang B, Wan W, Zhai W, Xu L, Hu K, and Xiao P (2016) Metabolomics reveals the protective of dihydromyricetin on glucose homeostasis by enhancing insulin sensitivity. Sci Rep 6:36184.

Leavenworth JW, Tang X, Kim HJ, Wang X, and Cantor H (2013) Amelioration of arthritis through mobilization of peptide-specific CD8+ regulatory $\mathrm{T}$ cells. J Clin Invest 123:1382-1389.

Lefêvre S, Knedla A, Tennie C, Kampmann A, Wunrau C, Dinser R, Korb A, Schnäker EM, Tarner IH, Robbins PD, et al. (2009) Synovial fibroblasts spread rheumatoid arthritis to unaffected joints. Nat Med 15:1414-1420.

Li N, Xu Q, Liu Q, Pan D, Jiang Y, Liu M, Liu M, Xu H, and Lin C (2017) Leonurine attenuates fibroblast-like synoviocyte-mediated synovial inflammation and joint destruction in rheumatoid arthritis. Rheumatology (Oxford) 56:1417-1427.

Li T, Wong VKW, Jiang ZH, Jiang SP, Liu Y, Wang TY, Yao XJ, Su XH, Yan FG, Liu $\mathrm{J}$, et al. (2015) Mutation of cysteine 46 in IKK-beta increases inflammatory responses. Oncotarget 6:31805-31819.

Liu H and Pope RM (2003) The role of apoptosis in rheumatoid arthritis. Curr Opin Pharmacol 3:317-322.

Liu Y, Pan YF, Xue YQ, Fang LK, Guo XH, Guo X, Liu M, Mo BY, Yang MR, Liu F, et al. (2017) uPAR promotes tumor-like biologic behaviors of fibroblast-like synoviocytes through PI3K/Akt signaling pathway in patients with rheumatoid arthritis. Cell Mol Immunol 15:171-181.

McInnes IB and Schett G (2007) Cytokines in the pathogenesis of rheumatoid arthritis. Nat Rev Immunol 7:429-442.

Miagkov AV, Kovalenko DV, Brown CE, Didsbury JR, Cogswell JP, Stimpson SA, Baldwin AS, and Makarov SS (1998) NF-kappaB activation provides the potential link between inflammation and hyperplasia in the arthritic joint. Proc Natl Acad Sci USA 95:13859-13864.
Mor A, Abramson SB, and Pillinger MH (2005) The fibroblast-like synovial cell in rheumatoid arthritis: a key player in inflammation and joint destruction. Clin Immunol 115:118-128.

Müller-Ladner U, Pap T, Gay RE, Neidhart M, and Gay S (2005) Mechanisms of disease: the molecular and cellular basis of joint destruction in rheumatoid arthritis. Nat Clin Pract Rheumatol 1:102-110.

Murphy G and Nagase H (2008) Progress in matrix metalloproteinase research. Mol Aspects Med 29:290-308.

Nieto FR, Clark AK, Grist J, Chapman V, and Malcangio M (2015) Calcitonin generelated peptide-expressing sensory neurons and spinal microglial reactivity contribute to pain states in collagen-induced arthritis. Arthritis Rheumatol 67:1668-1677.

Okazaki Y, Sawada T, Nagatani K, Komagata Y, Inoue T, Muto S, Itai A, and Yamamoto K (2005) Effect of nuclear factor-kappaB inhibition on rheumatoid fibroblast-like synoviocytes and collagen induced arthritis. J Rheumatol 32:1440-1447.

Pap T, Nawrath M, Heinrich J, Bosse M, Baier A, Hummel KM, Petrow P, Kuchen S, Michel BA, Gay RE, et al. (2004) Cooperation of Ras- and c-Myc-dependent pathways in regulating the growth and invasiveness of synovial fibroblasts in rheumatoid arthritis. Arthritis Rheum 50:2794-2802.

Qiao H, Wang TY, Yu ZF, Han XG, Liu XQ, Wang YG, Fan QM, Qin A, and Tang TT (2016) Structural simulation of adenosine phosphate via plumbagin and zoledronic acid competitively targets JNK/Erk to synergistically attenuate osteoclastogenesis in a breast cancer model. Cell Death Dis 7:e2094.

Qu Z, Garcia CH, O’Rourke LM, Planck SR, Kohli M, and Rosenbaum JT (1994a) Local proliferation of fibroblast-like synoviocytes contributes to synovial hyperplasia. Results of proliferating cell nuclear antigen/cyclin, c-myc, and nucleolar organizer region staining. Arthritis Rheum 37:212-220.

Rico MC, Castaneda JL, Manns JM, Uknis AB, Sainz IM, Safadi FF, Popoff SN, and Dela Cadena RA (2007) Amelioration of inflammation, angiogenesis and CTGF expression in an arthritis model by a TSP1-derived peptide treatment. $J$ Cell Physiol 211:504-512.

Shang-ling Zhu, Jian-lin Huang, Wei-xiang Peng, Dan-chun Wu, et al. (2017) Inhibition of smoothened decreases proliferation of synoviocytes in rheumatoid arthritis. Cell Mol Immunol 14:214-222.

Tak PP and Bresnihan B (2000) The pathogenesis and prevention of joint damage in rheumatoid arthritis: advances from synovial biopsy and tissue analysis. Arthritis Rheum 43:2619-2633.

Takemura S, Braun A, Crowson C, Kurtin PJ, Cofield RH, O'Fallon WM, Goronzy JJ, and Weyand CM (2001) Lymphoid neogenesis in rheumatoid synovitis. J Immunol 167:1072-1080.

van den Brand BT, Abdollahi-Roodsaz S, Vermeij EA, Bennink MB, Arntz OJ, Rothlin CV, van den Berg WB, and van de Loo FA (2013) Therapeutic efficacy of Tyro3, Axl, and Mer tyrosine kinase agonists in collagen-induced arthritis. Arthritis Rheum 65:671-680.

Vincenti MP, Coon CI, and Brinckerhoff CE (1998) Nuclear factor kappaB/p50 activates an element in the distal matrix metalloproteinase 1 promoter in interleukin1beta-stimulated synovial fibroblasts. Arthritis Rheum 41:1987-1994.

Wang W, Qu M, Xu L, Wu X, Gao Z, Gu T, Zhang W, Ding X, Liu W, and Chen YL (2016) Sorafenib exerts an anti-keloid activity by antagonizing TGF- $\beta /$ Smad and MAPK/ERK signaling pathways. J Mol Med (Berl) 94:1181-1194.

Wu Y, Bai J, Zhong K, Huang Y, and Gao H (2017) A dual antibacterial mechanism involved in membrane disruption and DNA binding of 2R,3R-dihydromyricetin from pine needles of Cedrus deodara against Staphylococcus aureus. Food Chem 218:463-470.

Xiao Y, Sun M, Zhan Z, Ye Y, Huang M, Zou Y, Liang L, Yang X, and Xu H (2014) Increased phosphorylation of ezrin is associated with the migration and invasion of fibroblast-like synoviocytes from patients with rheumatoid arthritis. Rheumatology (Oxford) 53:1291-1300.

Xin W, Huang C, Zhang X, Xin S, Zhou Y, Ma X, Zhang D, Li Y, Zhou S, Zhang D, et al. (2014) Methyl salicylate lactoside inhibits inflammatory response of fibroblast-like synoviocytes and joint destruction in collagen-induced arthritis in mice. Br J Pharmacol 171:3526-3538.

Yang Y, Dong Q, and Li R (2017) Matrine induces the apoptosis of fibroblast-like synoviocytes derived from rats with collagen-induced arthritis by suppressing the activation of the JAK/STAT signaling pathway. Int $J$ Mol Med 39:307-316.

Ye L, Wang H, Duncan SE, Eigel WN, and O'Keefe SF (2015) Antioxidant activities of vine tea (Ampelopsis grossedentata) extract and its major component dihydromyricetin in soybean oil and cooked ground beef. Food Chem 172:416-422.

Yeo L, Adlard N, Biehl M, Juarez M, Smallie T, Snow M, Buckley CD, Raza K, Filer A, and Scheel-Toellner D (2016) Expression of chemokines CXCL4 and CXCL7 by synovial macrophages defines an early stage of rheumatoid arthritis. Ann Rheum Dis 75:763-771.

Zhao L, Cai C, Wang J, Zhao L, Li W, Liu C, Guan H, Zhu Y, and Xiao J (2017) Dihydromyricetin protects against bone loss in ovariectomized mice by suppressing osteoclast activity. Front Pharmacol 8:928

Zhou HF, Yan H, Pan H, Hou KK, Akk A, Springer LE, Hu Y, Allen JS, Wickline SA and Pham CT (2014) Peptide-siRNA nanocomplexes targeting NF-кB subunit p65 suppress nascent experimental arthritis. J Clin Invest 124:4363-4374.

Address correspondence to: Dr. Ting-Yu Wang, Department of Pharmacy, Shanghai Ninth People's Hospital, Shanghai Jiao Tong University School of Medicine, 639 Zhizaoju Rd., Shanghai 200011, China. E-mail: drtywang@ 163.com 